Daimon. Revista Internacional de Filosofía, n ${ }^{\circ} 85$ (2022), pp. 67-82

ISSN: 1130-0507 (papel) y 1989-4651 (electrónico)

http://dx.doi.org/10.6018/daimon.398141

Licencia Creative Commons Reconocimiento-NoComercial-SinObraDerivada 3.0 España (texto legal). Se pueden copiar, usar, difundir, transmitir y exponer públicamente, siempre que: i) se cite la autoría y la fuente original de su publicación (revista, editorial y URL de la obra); ii) no se usen para fines comerciales; iii) se mencione la existencia y especificaciones de esta licencia de uso. (c) (1) () $\odot$

\title{
Panpsiquismo de construcción
}

\section{Building panpsychism}

\author{
ESTEBAN DIEGO ORTIZ MEDINA*
}

\begin{abstract}
Resumen: El principal problema del panpsiquismo es el problema de la combinación. Como solución se distinguen dos tipos de panpsiquismo: el panpsiquismo constitutivo y el panpsiquismo emergentista. Pero esta distinción opera sobre la distinción entre fundación y causalidad. Sin embargo, se verán razones para considerar que causalidad y fundación no son distintos. De modo que la distinción entre los anteriores panpsiquismos no es tal, posibilitando la postulación de un nuevo tipo de panpsiquismo: el panpsiquismo de construcción. También, se trazará una solución para el problema de la combinación desde el punto de vista del panpsiquismo de construcción. Palabras claves: Problema de la combinación, panpsiquismo constitutivo, panpsiquismo emergentista, identidad, causalidad, fundación.
\end{abstract}

\begin{abstract}
The main problem of panpsychism is the combination problem. As a solution, two types of panpsychism are distinguished: constitutive panpsychism and emergent panpsychism. But this distinction operates on the distinction between grounding and causality. However, there will be reasons to consider that causality and grounding are not different. So, the distinction between panpsychisms is not such, enabling the postulation of a new kind of panpsychism: the building panpsychism. Also, a solution for the combination problem from the point of view of building panpsychism will be drawn up.
\end{abstract}

Keywords: Combination problem, constitutive panpsychism, emergent panpsychism, identity, causality, grounding.

El principal problema del panpsiquismo es el problema de la combinación. En vista a esto, propondremos un nuevo tipo de panpsiquismo: el panpsiquismo de construcción. A partir de esta posición trazaremos una solución al anterior problema, que tiene mayor mérito que las otras soluciones propuestas desde el panpsiquismo. Para lograr el objetivo estructuraremos el trabajo del siguiente modo.

Recibido: 03/10/2019. Aceptado: 20/10/2020.

* Estudiante del Doctorado en Filosofía de la Universidad de Buenos Aires. Becario CONICET Latinoamericanas con lugar de trabajo en el IIF-SADAF-CONICET. Líneas actuales de investigación: filosofía de la mente (panpsiquismo y el problema de la combinación) y metafísica (metafísica de la ciencia, relaciones de dependencia ontológica y fundamentalidad). Publicaciones recientes: Ortiz, E. (2018). Sartre: una teoría autorepresentacional de la conciencia. Revista de Humanidades de Valparaíso, 11, 115-137, doi: http://dx.doi. org/10.22370/rhv.2018.11.850. Ortiz, E. (2019). Conciencia pre-reflexiva y la tesis de la identidad experiencia/ experimentador. Límite (Arica), 14, 12, doi: https://dx.doi.org/10.4067/s0718-50652019000100212. Correo electrónico: edeoeme@gmail.com 
En primer lugar, presentaremos el panpsiquismo y el problema de la combinación. Comúnmente, como respuesta a este problema, se distinguen dos tipos de panpsiquismo: panpsiquismo constitutivo y panpsiquismo emergentista. La distinción entre éstos opera sobre la distinción entre fundación y causalidad. Dado que las anteriores son tratadas como distintas, estos panpsiquismos son considerados como distintos (II). Pero tal distinción puede ser considerada falsa. Hay razones para considerar que fundación y causalidad son idénticas, y para esto se mostrarán las profundas semejanzas estructurales entre fundación y causalidad y el fallo de criterios que pretenden probar una distinción entre ambas (III). De este modo, la distinción entre panpsiquismo constitutivo y panpsiquismo emergentista es una distinción inadecuada porque está basada en una falsa distinción. De esta forma, el panpsiquismo de construcción surge como opción. La solución al problema de la combinación propuesta desde este nuevo panpsiquismo se basa en una sola y misma relación de dependencia ontológica unificada: la relación de construcción. El panpsiquismo de construcción ofrece una sola y misma relación de construcción unificada entre entidades de diferentes niveles y tiempos. A medida que avance el trabajo la definición de panpsiquismo de construcción y su solución al problema de la combinación será enriquecida (IV).

A partir de allí, evaluaremos la solución propuesta y a partir de esta evaluación, postularemos principios generales de construcción (V). La postulación de principios generales de construcción no es arbitraria porque en vista a que toda explicación requiere de un vínculo que conecte las fuentes a los resultados, éstos pueden considerarse como tales vínculos. Posteriormente, definiremos y caracterizaremos los principios generales de construcción como generalizaciones no-accidentales que logran un patrón explicativo general. A continuación, lo aplicaremos al panpsiquismo de construcción, dando como resultado la última definición en este trabajo del panpsiquismo de construcción y su solución al problema de la combinación (VI). Finalmente, se mostrarán razones para considerar que, en relación con los otros panpsiquismos, el panpsiquismo de construcción tiene mayor mérito (VII).

El panpsiquismo de construcción es un ejercicio de metafísica aplicada, se inspira en los actuales tratamientos de la literatura sobre dependencia ontológica, y aplica tales tratamientos de una manera novedosa al panpsiquismo y al problema de la combinación.

\section{II}

El panpsiquismo es la teoría que postula a la conciencia fenoménica (o experiencia o propiedades fenoménicas, pueden usarse de manera intercambiable) como ubicua. ${ }^{1}$ De algún modo la experiencia está involucrada con todo en la realidad, está presente en entidades donde inicialmente no se ubicaría (sin que ello implique que todo sea fenoménicamente consciente). Pero además, postula a la conciencia fenoménica como un constituyente fundamental de la realidad: la experiencia es ontológicamente independiente, no depende de otra cosa. Por lo tanto, no es una propiedad (fuertemente) emergente. ${ }^{2}$ De este modo, la

1 La conciencia fenoménica es entendida en el siguiente sentido: una entidad es fenoménicamente consciente si hay algo que es cómo es ser esa entidad. Una entidad es fenoménicamente consciente si hay algo que es cómo ser desde dentro ser esa entidad (Nagel (2003)).

2 Sobre la emergencia véase: van Gulick (2001). Es posible distinguir entre dos tipos de emergencia. Emergencia débil y emergencia fuerte. Cuando una entidad de un nivel superior surge de un nivel inferior sin que sea posi- 
experiencia no es reducible a o explicable en términos de constituyentes más fundamentales de la realidad enteramente no-experienciales.

Usualmente se considera a las entidades microfísicas postuladas por la ciencia física como los constituyentes fundamentales de la realidad (Kim 2002, 53). Por lo tanto, el panpsiquismo adscribe subjetividad a las entidades fundamentales microfísicas, es decir, las entidades fundamentales microfísicas serían los sujetos de experiencia fundamentales (hay una conjunción entre ubicuidad y fundamentalidad). Ser sujeto de experiencia denota la experiencia de ser un locus interno de conciencia solo accesible desde el respectivo punto de vista del sujeto de experiencia. Un sujeto de experiencia sería "esencialmente ser para sí mismo, en el sentido familiar de esta frase según la cual para que un ser sea 'para sí mismo' es que hay algo que es cómo es ser ese ser, experiencialmente" (Strawson 2017, 80). Para el panpsiquismo, hay algo que es cómo es ser una entidad fundamental microfísica que se agota enteramente en ser un sujeto de experiencia.

Para explicar la conciencia fenoménica de una entidad dependiente (es decir, nofundamental) el panpsiquismo postula que la experiencia es un constituyente fundamental de la realidad. Así, la conciencia de un sujeto de experiencia dependiente, un ser humano (por ejemplo), está de algún modo basada en las propiedades fenoménicas de las entidades fundamentales microfísicas.

El espíritu del panpsiquismo es de continuidad y uniformidad. Téngase en cuenta el siguiente dictum: la realidad no da saltos (Leibniz 1981, 56). La realidad es continua y uniforme. Si no se tuviese en cuenta al panpsiquismo, existiría un hiato en la realidad entre una porción enteramente no-experiencial y una porción que implica la experiencia. Así los sujetos de experiencia dependientes se basarían en algo enteramente no-experiencial, lo cual introduce discontinuidad, que rompe con la uniformidad de la realidad. Pero si se tiene en cuenta al panpsiquismo, no existiría el mentado hiato. Sujetos de experiencia fundamentales y sujetos de experiencia dependientes son ambos igualmente sujetos de experiencia. Hay continuidad y uniformidad en la realidad. Los sujetos de experiencia dependientes se moldean desde propiedades fenoménicas preexistentes y no desde propiedades enteramente no-experienciales. De modo que se producen formas más complejas de experiencia y no propiedades totalmente nuevas (propiedades (fuertemente) emergente).

A pesar de que a primera vista el panpsiquismo suena contraintuitivo, tiene grandes ventajas. Hace frente al problema difícil de la conciencia (Chalmers (1995)), cierra la brecha explicativa (Levine (1983)). Además, puede plantearse como una respuesta al problema de la interacción mentelcuerpo (Chalmers 2010, 133-134). Pero como toda

\footnotetext{
ble, ni siquiera en principio, deducir la primera del segundo, es el caso la emergencia fuerte. La entidad fuertemente emergente tiene, al menos, un poder causal que no es idéntico a ningún poder causal del nivel inferior. Cuando una entidad de un nivel superior surge de un nivel inferior y la primera es inesperada en relación con los principios nómicos que rigen al nivel inferior, es el caso la emergencia débil. La entidad débilmente emergente tiene un subconjunto propio no-vacío de poderes causales del nivel inferior. Ambos casos de emergencia son similares porque caracterizan los poderes de las entidades emergentes con relación a los poderes causales de las entidades base. Ambos casos de emergencia son disímiles porque la emergencia débil es compatible con el fisicalismo (no-reductivo). En cambio, la emergencia fuerte no lo es. Si esta última es el caso, y existe una entidad que no es deducible desde una distribución de campos y partículas, entonces el fisicalismo es rechazado por incompleto. Para mayor detalle véase: Chalmers (2006), Wilson, J. (2016).
} 
teoría arrastra problemas. El principal problema del panpsiquismo es el problema de la combinación (James (1983)). ${ }^{3}$

Aun cuando se tenga la idea de que la conciencia de un sujeto de experiencia dependiente está de algún modo basada en las propiedades fenoménicas de las entidades fundamentales microfísicas, es tremendamente problemático dar una explicación de esto. ¿Qué tipo de relación combinatoria deben entablar tales entidades para que sus respectivas micro-experiencias se combinen, y como resultado de esa combinación se genere una entidad macrofísica con su respectiva macro-experiencia? Este problema se hace más agudo si no se observa necesidad de combinación cuando existe $n$ cantidad de sujetos de experiencia y se agrega uno más.

Puesto que las micro-experiencias de las entidades fundamentales microfísicas se presuponen como característicamente granulares y simples, y, en cambio, las macro-experiencias de una entidad macrofisica, como las de un ser humano, característicamente instancian distintos grados de complejidad, es muy difícil explicar el paso de lo pequeño a lo grande, si lo pequeño se considera como explanans. El panpsiquismo tiene su propia brecha explicativa.

De manera alternativa, la brecha explicativa del panpsiquismo - el problema de la combinación - puede entenderse como la pregunta por las características especiales de la composición fenoménica (the special phenomenal composition question). Es decir, ¿bajo qué condiciones las propiedades fenoménicas de las entidades fundamentales microfísicas se combinan para formar un sujeto de experiencia dependiente? (Goff 2011, 136; cfr. van Inwagen 1990).

La respuesta desde el panpsiquismo para el problema de la combinación puede darse de dos maneras. Como panpsiquismo constitutivo (constitutive panpsychism) o como panpsiquismo emergentista (emergent panpsychists):

- Panpsiquismo constitutivo $=_{\mathrm{df}}$ la conciencia de un sujeto de experiencia dependiente está fundada (grounded) en la experiencia de las entidades fundamentales microfísicas (Goff (2017), Roelofs (2014)). ${ }^{4}$

- Panpsiquismo emergentista $=_{\text {df }}$ la conciencia de un sujeto de experiencia dependiente emerge (débilmente) de la actividad causal de la experiencia de las entidades fundamentales microfísicas (Mørch (2014), Seager (2010)).

Entonces, según el panpsiquismo constitutivo la conciencia de un sujeto de experiencia dependiente lo es en virtud de la experiencia de las entidades fundamentales microfísicas. En cambio, para el panpsiquismo emergentista la conciencia de un sujeto de experiencia dependiente no está fundada en la experiencia de las entidades fundamentales microfísicas, es causalmente (débilmente) emergente de la experiencia de las entidades fundamentales microfísicas. Es decir, y de manera más específica, como hace notar Goff: "El [panpsiquismo] constitutivo sostiene que [la solución al problema de la combinación] toma la forma de una fundación constitutiva (constitutive grounding), mientras que el [panpsiquismo] emergentista sostiene que [la solución al problema de la combinación] es causal”. $(2017,173)$.

La distinción entre panpsiquismo constitutivo y panpsiquismo emergentista, en última instancia, opera sobre la distinción entre fundación y causalidad. Estos panpsiquismos son

3 Véase Chalmers (2017) para un desarrollo más detallado de qué es el problema de la combinación, sus variedades e implicancias.

4 Por fundación, véase más adelante. 
considerados como distintos porque las anteriores relaciones son tratadas como totalmente distintas (Bernstein (2016), Koslicki (2016), Wilson, J. (2014)). Pero es posible el tratamiento de la fundación a la imagen de la causalidad o de la causalidad a la imagen de la fundación (Alvarado (MS), Bennett (2011, 2017), Kivatinos (2017), Schaffer (2016), Wilson, A. (2018)). Con lo cual, tal distinción puede ser puesta en duda. Existen sistemáticas analogías, profundas semejanzas estructurales, entre fundación y causalidad que insinúan la identidad entre éstas.

Entonces si hay una relación de identidad entre fundación y causalidad la distinción entre panpsiquismo constitutivo y panpsiquismo emergentista es inadecuada. Lo cual permite proponer otro tipo de panpsiquismo, al que llamaremos panpsiquismo de construcción (building panpsychism) ${ }^{5}$ :

- (A1) Panpsiquismo de construcción = df la conciencia de un sujeto de experiencia dependiente está construida (built) a partir de la experiencia de las entidades fundamentales microfísicas.

\section{III}

Antes de desarrollar el panpsiquismo de construcción en mayor detalle, es necesario, primero, una mínima definición de fundación y causalidad, y segundo mostrar los motivos que insinúan la identidad entre ambas.

La fundación (grounding) (Fine (2001), Rosen (2010), Schaffer (2009)) se caracteriza como irreflexiva (no puede ocurrir que el hecho $x$ funda el hecho $x$, nada se fundaría a sí mismo); asimétrica (si el hecho $x$ funda el hecho $y$, no puede ocurrir que el hecho $y$ funde el hecho $x$. Está excluida la fundación circular); y transitiva (si el hecho $x$ funda el hecho $y, \mathrm{y}$ si el hecho $y$ funda el hecho $z$ entonces el hecho $x$ funda el hecho $z$, existiría una cadena de fundación). En vista a esto, la fundación sería un orden estricto parcial (Raven 2015, 327). Además, es posible caracterizarla como necesaria (si el hecho $x$ funda el hecho $y$ entonces, necesariamente, si el hecho $x$ es el caso, entonces el hecho $y$ es el caso); explicativa (si el hecho $x$ funda el hecho $y$ entonces el hecho $x$ (metafísicamente) explica el hecho y); no-monotónica (si el hecho $x$ funda el hecho $z$, no puede ocurrir que el hecho $x$ funda el hecho $z$ junto con otro hecho $y$. Está excluida la adición no justificada de hechos fundantes); es hiperintensional (hyperintensional) (salva veritate, términos correferentes no pueden ser sustituidos); y bienfundada (el hecho $x$ se basa en el hecho fundamental o él mismo es el hecho fundamental).

La causalidad (García Encinas (2018), Miguel (2019)) se entiende como una relación en la que están involucrados, como mínimo, dos relata. Causa y efecto. Ésta posee las siguientes características. Es asimétrica. Existe tanto una prioridad temporal de la causa sobre el efecto, es decir, la causa ocurre antes del efecto, como una conjunción constante, lo cual quiere decir que si una causa es el caso entonces un efecto es el caso. La causalidad es unidireccional. Entre causa y efecto existe contigüidad espaciotemporal. También, es irreflexiva porque ninguna causa se causa a sí misma. Además, es transitiva porque si el hecho $x$ causa el hecho $y$, y si el hecho $y$ causa el hecho $z$ entonces el hecho $x$ causa el hecho $z$, existe una cadena de causalidad. La causalidad es explicativa: si el hecho $x$ causa el hecho $y$ entonces

5 Tomamos como referencia la propuesta de Bennett sobre las relaciones de construcción, pero no nos comprometemos con (todas) las consecuencias del aparataje conceptual de la autora. 
el hecho $x$ (causalmente) explica el hecho $y$. Por último, bajo específicas y determinadas circunstancias, se puede plantear que la causalidad implica cierto grado mínimo de necesi$d a d$, en el sentido de que la causa es necesitador (necessitates) de su efecto (Heil 2015, 117).

Las (relevantes) sistemáticas analogías, profundas semejanzas estructurales, entre fundación y causalidad serían las siguientes:

- Ambas relaciones comparten varios rasgos formales (como la irreflexividad, la asimetría y la transitividad). Ambas parecen ser órdenes estrictos parciales (Bennett (2017), Kivatinos (2017), Schaffer (2016), Wilson, A. (2018)).

- Ambas poseen un carácter productivo, es decir, $x$ produciría o generaría $y$. Dicho de otro modo: para todo $x$ e $y$, Rxy permite afirmaciones generativas en el sentido de que $y$ existe o se da en virtud de $x$ (Bennett (2017), Kivatinos (2017), Schaffer (2016)).

- Ambas están respaldadas por generalizaciones no-accidentales. Son relaciones nómicas y delimitan formas específicas de necesidad (Kivatinos (2017), Schaffer (2016), Wilson, A. (2018)).

- Ambas relaciones pueden citarse de manera informativa en explicaciones, es decir, ambas relaciones respaldan (back) la explicación. Por lo tanto, subyacen a la estructura explicativa de la realidad (Kivatinos (2017), Schaffer (2016), Wilson, A. (2018)).

- Ambas relaciones imponen una estructura metafísica en el sentido de que ordenan metafísicamente los contenidos de la realidad (Kivatinos (2017)). ${ }^{6}$

Es claro que fundación y causalidad comparten varios rasgos formales, basta con tener en cuenta las definiciones anteriores. Las otras analogías señaladas serán desarrolladas en detalle a medida que avance este trabajo. Será manifiesto que el panpsiquismo de construcción dado su carácter productivo, a través de su conexión con la explicación, al estar respaldado por generalizaciones no-accidentales, brindará una solución al problema de la combinación.

Por otro lado, Wilson, A. (MS) recaba una serie de criterios que se proponen para mostrar la distinción entre fundación y causalidad. Nombraremos algunos:

- Criterio de distinción: La causalidad relaciona distintos tipos de entidades, en cambio la fundación relaciona entidades de un mismo tipo.

- Criterio de fundamentalidad: La causalidad no tendría relación alguna con la fundamentalidad, en cambio si el hecho $x$ funda el hecho $y$ entonces el primero es más fundamental que el segundo.

- Criterio temporal: La causalidad es diacrónica, en cambio la fundación sincrónica.

Pero cada criterio falla. Por ejemplo, el criterio temporal falla porque si nos movemos más allá de los casos paradigmáticos (arrojar una roca es temporalmente prioritario a quebrar una ventana, Sócrates es ontológicamente prioritario a \{Sócrates\}) éste empieza a parecer

6 Otras analogías y semejanzas (menos relevantes en el contexto de este trabajo) entre fundación y causalidad son las siguientes: varios rasgos formales que comparten han sido cuestionados por medio de casos estructuralmente similares y las respuestas a estos cuestionamientos son análogas. Ambas relaciones pueden ser fructíferamente regimentadas por el mismo formalismo matemático, i.e. modelos de ecuaciones estructurales (vid. Halpern (2000), Pearl (2009)), sin diferencia alguna en la forma en cómo son tratados por este formalismo. Fundación y causalidad están estrechamente asociadas a distintivos patrones de dependencia contrafactual, en consecuencia, ambas relaciones son diagnosticables vía contrafácticos. 
dudoso. Según Wilson, A., una razón para rechazar el criterio temporal es que postular una fundación diacrónica es coherente desde el punto de vista metafísico. La pertenencia de un individuo a una clase natural, que depende de la historia causal de tal clase natural, podría considerarse un caso de ésta. Lo que se necesita para ser un ser humano es descender del homo sapiens, es decir, depender temporalmente de la historia causal del homo sapiens. Ser un ser humano está fundado en la ocurrencia de algunos eventos pasados: aquellos que constituyen el árbol filogenético de la correspondiente clase natural. Un perfecto duplicado de un ser humano formado por puro azar ex nihilo no podría ser correctamente considerado como tal porque no compartiría la historia causal que lo relaciona a la clase natural (MS, 16-20).

El fallo de los criterios que pretenden mostrar la distinción entre fundación y causalidad, y la existencia de profundas semejanzas entre éstas, nos sugiere que ambas están más íntimamente relacionadas de lo que pensamos, y que tal íntima relación es la que de alguna manera explica las semejanzas y los fallos. Podría pensarse que fundación y causalidad, si bien numéricamente distintas, se encuentran íntimamente relacionadas al ser distintas especies de un mismo género de dependencia ontológica más neutral y abstracto. Pero según una serie de criterios (Kivatinos 2017, vii) es la identidad entre fundación y causalidad la que explica lo anterior de mejor manera, es decir, por qué los criterios de distinción antes nombrados fallan y por qué existen profundas semejanzas estructurales entre ellas. Por ejemplo, la identidad da cuenta de la conexión entre explicación causal y explicación metafísica. No está del todo claro que la distinción entre explicación causal y explicación metafísica se sostenga, tal distinción puede ser plausiblemente negada (Kivatinos 2017, 61-69). Por lo tanto, solo hay un tipo de explicación. La identidad se hace más comprensible la negación de la distinción entre estos tipos de explicación, lo que no se lograría si consideramos a la fundación y a la causalidad como distintas. En consecuencia, la idea estándar según la cual éstas son totalmente distintas queda puesta en duda.

Además, puede excluirse la idea según la cual fundación y causalidad son distintas especies de un mismo género de dependencia ontológica más neutral y abstracto porque la identidad es conceptualmente más parsimoniosa (Kivatinos 2017, 57-61 y Wilson, A. 2018, 2). La parsimonia conceptual es una cuestión de la cantidad de conceptos que se necesitan para entender las relaciones anteriores. Al ser distintas especies de un mismo género de dependencia ontológica, fundación y causalidad son entendidas bajo diferentes conceptos: dos conceptos de especie más el concepto de género. Alternativamente, al identificar fundación y causalidad, no es necesario lo anterior, solo se necesita un concepto, el concepto de "construcción", bajo el cual se entienden fundación y causalidad, de modo que es posible prescindir de los anteriores conceptos. Por lo tanto, al ser entendida bajo menos conceptos, la relación de identidad es más conceptualmente parsimoniosa que la relación género/especie, así que es preferible ésta sobre la segunda. Como puede observarse, la cuestión terminológica es simplemente una consecuencia de la identidad entre fundación y causalidad.

Otra consecuencia de la identidad entre fundación y causalidad es el monismo con respecto a las relaciones de dependencia ontológica (Berker (2018)). Es decir, hay una sola y misma relación de dependencia ontológica fuertemente unificada. La relación de construcción. No habría una diferencia sustancial en la naturaleza misma de la relación (por lo tanto, no serían tres relaciones ontológica y numéricamente distintas) si ésta ordena metafísicamente los contenidos de la realidad a través del tiempo (lo que usualmente se entiende 
como causalidad), ordena metafísicamente los contenidos de la realidad a través de niveles ontológicos (lo que usualmente se entiende como fundación) u ordena metafísicamente los contenidos de la realidad tanto a través del tiempo como a través de niveles ontológicos. Ni tampoco serían relaciones ontológica y numéricamente distintas si ésta ordena metafísicamente entidades diferentes (e.g. la relación que se sostiene entre entidades no-biológicas y entidades biológicas). No importa la constitución intrínseca de sus relata o el orden metafísico impuesto, la relación de construcción es solo relación de construcción en todas sus instancias. De este modo pueden desestimarse la relación de fundación y la relación de causalidad porque se basa en una distinción falsa.

\section{IV}

Decíamos, si hay una relación de identidad entre fundación y causalidad entonces la distinción entre panpsiquismo constitutivo y panpsiquismo emergentista es inadecuada. Estos panpsiquismos son considerados como distintos porque, de manera estándar, las relaciones ofrecidas (fundación y causalidad) como respuesta al problema de la combinación son tratadas como distintas. Pero como ya vimos, fundación y causalidad pueden considerarse como idénticas. La distinción entre estas relaciones no es tal, y por lo tanto, tampoco lo será la distinción entre estos dos tipos de pansiquismo. No hay una relación de dependencia ontológica que ordena metafísicamente los contenidos de la realidad a través del tiempo diferente de una relación de dependencia ontológica que ordena metafísicamente los contenidos de la realidad a través de niveles ontológicos, en virtud de la cual el panpsiquismo emergentista pueda ser considerado diferente del panpsiquismo constitutivo. En cambio, podemos afirmar que hay una sola y misma relación de dependencia ontológica unificada, la relación de construcción, que da lugar al panpsiquismo de construcción. Por esto el panpsiquismo de construcción aparece como opción; el panpsiquismo de construcción sería el resultado de la identidad de los anteriores. Pero sobre todo, es una opción mejor que las anteriores porque presenta una solución al problema de la combinación.

Con las profundas semejanzas estructurales mencionadas anteriormente en mente, podemos decir que en el panpsiquismo de construcción se funda en la idea de producción: las propiedades fenoménicas de algunas entidades fundamentales microfísicas construirían un sujeto de experiencia dependiente. Así, podemos presuponer que una de las principales características de la solución provista por este panpsiquismo será la construcción de un sujeto de experiencia dependiente por medio del arreglo (arrangement) dinámico temporal (horizontalmente extendido a través del tiempo) y ontológico (verticalmente extendido a través de niveles ontológicos) de las propiedades fenoménicas de un número $n$ de entidades fundamentales microfísicas. El panpsiquismo de construcción ofrece una sola y misma relación de construcción unificada entre entidades de diferentes niveles y tiempos.

A esta altura, es el conjunto de las propiedades fenoménicas de un número $n$ de entidades fundamentales microfísicas las que construyen a un sujeto de experiencia dependiente, ninguna de ellas es suficiente individualmente. Parece poco probable que una o un conjunto reducido de entidades fundamentales microfísicas construyan un sujeto de experiencia dependiente, como un ser humano, debido a lo mencionado párrafos atrás. Las micro-experiencias de las entidades fundamentales microfísicas se presuponen como característicamente granulares y simples. Con 
lo cual, para construir la macro-experiencia de una entidad macrofisica, que típicamente instancia distintos grados de complejidad, no parece errado pensar que se requeriría de un número indeterminado de entidades fundamentales microfísicas con sus respectivas experiencias en un determinado tipo de relación de producción ontológica. Entonces, un sujeto de experiencia dependiente es construido por el arreglo dinámico colectivo de las propiedades fenoménicas de un número $n$ de entidades fundamentales microfísicas.

Por ahora, la solución al problema de la combinación desde el punto de vista del panpsiquismo de construcción tomará la siguiente forma.

Considérese la siguiente situación. Sea la porción $(\Psi)$ de un $\alpha$-nivel más fundamental de la realidad, en el cual hay $n$ propiedades fenoménicas $\left(\mathrm{C}^{\alpha_{1}}, \mathrm{C}^{\alpha_{2}, \mathrm{C}^{\alpha}}, \ldots, \mathrm{C}^{\alpha}{ }_{n}\right)$ y $n$ entidades fundamentales microfísicas $\left(\mathrm{m}^{\alpha_{1}}, \mathrm{~m}^{\alpha_{2}}, \mathrm{~m}^{\alpha_{3}}, \ldots, \mathrm{m}^{\alpha}{ }_{n}\right)$ en relaciones de instanciación $\left(\mathrm{C}^{\alpha_{1}}\left(\mathrm{~m}^{\alpha_{1}}\right), \mathrm{C}^{\alpha_{2}}\left(\mathrm{~m}^{\alpha}{ }_{2}\right), \mathrm{C}^{\alpha_{3}}\left(\mathrm{~m}^{\alpha_{3}}\right), \ldots, \mathrm{C}^{\alpha}\left(\mathrm{m}^{\alpha}{ }_{n}\right)\right)$ en el tiempo $\left(\mathrm{t}_{1}\right)$. En tal $\alpha$-nivel podría ser el caso que el arreglo dinámico colectivo horizontalmente extendido a través del tiempo de


$\ldots, \mathrm{C}_{n+}\left(\mathrm{m}^{\alpha+}\right)$ en el tiempo $\left(\mathrm{t}_{2}\right)$. Y luego, podría ser el caso que el arreglo dinámico colectivo verticalmente extendido a través de niveles ontológicos de $\mathrm{C}^{\alpha_{1+}}\left(\mathrm{m}^{\alpha_{1+}}\right), \mathrm{C}^{\alpha_{2+}}\left(\mathrm{m}^{\alpha_{2+}}\right), \mathrm{C}^{\alpha_{3+}}\left(\mathrm{m}^{\alpha_{3+}}\right)$, $\ldots, \mathrm{C}_{n+}\left(\mathrm{m}^{\alpha}{ }_{n+}\right)\left(\mathrm{t}_{2}\right)$ produzca un sujeto de experiencia dependiente $\left(\mathrm{C}^{\beta_{1 *}}\left(\mathrm{~s}^{\beta_{1 *}}\right)\right)$ en el mismo tiempo $\left(\mathrm{t}_{2}\right)$. Correspondiente a la porción ( $\left.\Psi^{\prime}\right)$ de un $\beta$-nivel menos fundamental de la realidad (cfr. Humphreys (1997)). ${ }^{7}$

Ahora, con la transitividad de la relación de construcción en mente, el panpsiquismo de construcción establece que $\mathrm{C}^{\alpha}{ }_{1}\left(\mathrm{~m}^{\alpha}{ }_{1}\right), \mathrm{C}^{\alpha_{2}}\left(\mathrm{~m}^{\alpha}{ }_{2}\right), \mathrm{C}^{\alpha_{3}}\left(\mathrm{~m}^{\alpha}\right), \ldots, \mathrm{C}^{\alpha}{ }_{n}\left(\mathrm{~m}^{\alpha}{ }_{n}\right)\left(\mathrm{t}_{1}\right)$ construye $\mathrm{C}^{\beta_{1 *}}\left(\mathrm{~s}^{\beta_{1 *}}\right)$ $\left(\mathrm{t}_{2}\right)$. La transitividad es una propiedad de la relación de construcción por lo tanto debe haber una sola y misma relación de construcción unificada que la instancie (cfr. Kivatinos 2017, 70-74). Para el panpsiquismo de construcción, la relación anterior es una sola y misma relación de construcción unificada que ordena los contenidos de la realidad tanto de manera horizontalmente extendida a través del tiempo como de manera verticalmente extendida a través de niveles ontológicos.

Es decir, el arreglo dinámico colectivo de $\mathrm{C}^{\alpha}{ }_{1}\left(\mathrm{f}^{\alpha}{ }_{1}\right), \mathrm{C}^{\alpha}{ }_{2}\left(\mathrm{f}^{\alpha}{ }_{2}\right), \mathrm{C}^{\alpha}{ }_{3}\left(\mathrm{f}^{\alpha}{ }_{3}\right), \ldots, \mathrm{C}^{\alpha}{ }_{\mathrm{n}}\left(\mathrm{f}^{\alpha_{n}}\right)\left(\mathrm{t}_{1}\right)$, involucrado en una relación de construcción tanto horizontalmente extendida a través del tiempo como verticalmente extendida a través de niveles ontológicos, se despliega a $\mathrm{C}^{\alpha_{1+}}\left(\mathrm{m}^{\alpha_{1+}}\right)$, $\mathrm{C}^{\alpha}{ }{ }\left(\mathrm{m}^{\alpha}{ }_{2+}\right), \mathrm{C}^{\alpha}{ }_{3+}\left(\mathrm{m}^{\alpha}{ }_{3+}\right), \ldots, \mathrm{C}_{n^{+}}\left(\mathrm{m}^{\alpha}{ }_{n+}\right)\left(\mathrm{t}_{2}\right)$ hasta $\mathrm{C}^{\beta_{1 *}}\left(\mathrm{~s}^{\beta_{1 *}}\right)\left(\mathrm{t}_{2}\right)$, conectando $\mathrm{C}^{\alpha}{ }_{1}\left(\mathrm{~m}^{\alpha}{ }_{1}\right), \mathrm{C}^{\alpha_{2}}\left(\mathrm{~m}^{\alpha}{ }_{2}\right)$, $\mathrm{C}^{\alpha_{3}}\left(\mathrm{~m}^{\alpha}{ }_{3}\right), \ldots, \mathrm{C}^{\alpha}{ }_{n}\left(\mathrm{~m}^{\alpha}{ }_{n}\right)\left(\mathrm{t}_{1}\right)$ con $\mathrm{C}^{\beta_{1}}{ }^{\prime}\left(\mathrm{s}^{\beta_{1}}{ }^{\prime}\right)\left(\mathrm{t}_{2}\right)$. Esto es, el arreglo dinámico colectivo de $\mathrm{C}^{\alpha}{ }_{1}\left(\mathrm{f}^{\alpha}{ }_{1}\right)$, $\mathrm{C}^{\alpha_{2}}\left(\mathrm{f}_{2}{ }_{2}\right), \mathrm{C}^{\alpha_{3}}\left(\mathrm{f}_{3}\right), \ldots, \mathrm{C}^{\alpha_{n}}\left(\mathrm{f}_{\mathrm{n}} \mathrm{a}\right)\left(\mathrm{t}_{1}\right)$ en la porción $(\Psi)$ de un $\alpha$-nivel más fundamental de la realidad construye (en una sola y misma relación unificada) a $C^{\beta_{1}}{ }^{\prime \prime}\left(\mathrm{s}^{\beta_{1}}{ }^{\prime}\right)\left(\mathrm{t}_{2}\right)$ en la porción ( $\Psi$ ') de un $\beta$-nivel menos fundamental de la realidad.

Con lo dicho hasta ahora, podemos nuevamente definir al panpsiquismo de construcción:

- (A2) Panpsiquismo de construcción $=_{\mathrm{df}}$ la conciencia de un sujeto de experiencia dependiente está construida a partir del arreglo dinámico colectivo, tanto horizontalmente extendido a través del tiempo como verticalmente extendido a través de niveles ontológicos, de las propiedades fenoménicas de un número $n$ de entidades fundamentales microfísicas.

7 Seguimos la notación propuesta por Humphreys, pero no nos comprometemos con todas las consecuencias del aparataje conceptual del autor. 
Otro modo de entender el panpsiquismo de construcción se nos presenta al tener en cuenta la estrecha relación que hay entre la relación de construcción y la explicación, porque si el hecho $x$ construye el hecho $y$ entonces el hecho $x$ explica el hecho $y$. Es decir, esta relación respalda (back) la explicación. Con esto en mente, y desde la situación anterior, podemos establecer una similitud estructural en lo referente a la explicación y el panpsiquismo de construcción. La cual nos permite establecer que $C^{\alpha_{1}}\left(m^{\alpha_{1}}\right), C^{\alpha}{ }_{2}\left(m^{\alpha}{ }_{2}\right), C^{\alpha_{3}}\left(m^{\alpha}{ }_{3}\right)$, $\ldots, \mathrm{C}^{\alpha}\left(\mathrm{m}^{\alpha}\right)\left(\mathrm{t}_{1}\right)$ explica $\mathrm{C}^{\alpha_{1+}}\left(\mathrm{m}^{\alpha}{ }_{1+}\right), \mathrm{C}^{\alpha_{2+}}\left(\mathrm{m}^{\alpha_{2+}}\right), \mathrm{C}^{\alpha_{3+}}\left(\mathrm{m}^{\alpha_{3+}}\right), \ldots, \mathrm{C}_{n+}\left(\mathrm{m}^{\alpha}{ }_{n+}\right)\left(\mathrm{t}_{2}\right)$ y $\mathrm{C}^{\alpha_{1+}}\left(\mathrm{m}^{\alpha_{1+}}\right)$, $\mathrm{C}^{\alpha_{2+}}\left(\mathrm{m}^{\alpha}{ }_{2+}\right), \mathrm{C}^{\alpha_{3+}}\left(\mathrm{m}^{\alpha_{3+}}\right), \ldots, \mathrm{C}_{n+}\left(\mathrm{m}^{\alpha}{ }_{n+}\right)\left(\mathrm{t}_{2}\right)$ explica $\mathrm{C}^{\beta_{1 *}}\left(\mathrm{~s}^{\beta_{1 *}}\right)\left(\mathrm{t}_{2}\right)$.

Ahora, no parece errado que se trace una vía explicativa (explanatory path) entre


explica al tercero. Pero ¿qué relación de dependencia ontológica respalda tal vía explicativa? Schaffer ( $c f r .2016,89-90$ ) habla de una relación híbrida de dependencia ontológica, que involucra ciertos elementos de la fundación y de la causalidad. Es decir, la vía explicativa antes mencionada se obtiene por medio de una combinación entre las anteriores relaciones.

Pero eso sería desestimar todo lo establecido hasta ahora porque lo dicho se sustenta en la problemática distinción (previamente rechazada) entre fundación y causalidad, o más específicamente en la (supuesta) distinción entre explicación metafísica y explicación causal. Distinción que el propio Schaffer rechaza. Solo hay un tipo de explicación. La fórmula de Schaffer es: una explicación, varias relaciones de dependencia ontológica que la respaldan (fundación, causalidad, relación híbrida). Pero ¿por qué mejor no simplificar las cosas? ¿Por qué no considerar una relación de dependencia ontológica que no desestime todo lo dicho hasta ahora, que mantenga el rechazo a la distinción entre fundación y causalidad y a la distinción entre explicación metafísica y explicación causal? Así, la vía explicativa entre $\mathrm{C}^{\alpha_{1}}\left(\mathrm{~m}^{\alpha_{1}}\right), \mathrm{C}^{\alpha_{2}}\left(\mathrm{~m}^{\alpha_{2}}\right), \mathrm{C}^{\alpha_{3}}\left(\mathrm{~m}^{\alpha_{3}}\right), \ldots, \mathrm{C}^{\alpha}{ }_{n}\left(\mathrm{~m}^{\alpha}{ }_{n}\right)\left(\mathrm{t}_{1}\right)$ y $\mathrm{C}^{\beta_{1 *}}\left(\mathrm{~s}^{\beta_{1 *}}\right)\left(\mathrm{t}_{2}\right)$ no podría darse por medio de una combinación entre la relación de fundación y la relación de causalidad.

Entonces, si hay una vía explicativa entre $\mathrm{C}^{\alpha_{1}}\left(\mathrm{~m}^{\alpha_{1}}\right), \mathrm{C}^{\alpha_{2}}\left(\mathrm{~m}^{\alpha_{2}}\right), \mathrm{C}^{\alpha_{3}}\left(\mathrm{~m}^{\alpha_{3}}\right), \ldots, \mathrm{C}^{\alpha_{n}}\left(\mathrm{~m}^{\alpha}{ }_{n}\right)\left(\mathrm{t}_{1}\right)$ y


relación de construcción unificada tanto horizontalmente extendida a través del tiempo como verticalmente extendida a través de niveles ontológicos. Esto se refuerza al considerar que las explicaciones siguen la pista (tracks) de la relación de construcción (Kim (1994)). El punto es que si hay un tipo de explicación que sigue la pista de la relación de construcción es porque realmente hay un tipo de relación de dependencia ontológica que respalda la explicación (contra Schaffer) (vid. Wilhelm (2020)).

Con lo cual, si hay una vía explicativa que sigue la pista al despliegue de la relación de construcción desde $\mathrm{C}^{\alpha_{1}}\left(\mathrm{f}^{\alpha_{1}}\right), \mathrm{C}^{\alpha_{2}}\left(\mathrm{f}^{\alpha_{2}}\right), \mathrm{C}^{\alpha_{3}}\left(\mathrm{f}^{\alpha_{3}}\right), \ldots, \mathrm{C}^{\alpha_{n}}\left(\mathrm{f}^{\alpha_{n}}\right)\left(\mathrm{t}_{1}\right)$ a $\mathrm{C}^{\alpha_{1+}}\left(\mathrm{m}^{\alpha_{1+}}\right), \mathrm{C}^{\alpha_{2+}}\left(\mathrm{m}^{\alpha_{2+}}\right)$, $\mathrm{C}^{\alpha_{3+}}\left(\mathrm{m}^{\alpha_{3+}}\right), \ldots, \mathrm{C}_{n+}\left(\mathrm{m}^{\alpha+}\right)\left(\mathrm{t}_{2}\right)$ en la porción $(\Psi)$ de un $\alpha$-nivel más fundamental de la realidad hasta $\mathrm{C}^{\beta_{1 *}}\left(\mathrm{~s}^{\beta_{1 *}}\right)\left(\mathrm{t}_{2}\right)$ en la porción ( $\left.\Psi^{\prime}\right)$ de un $\beta$-nivel menos fundamental de la realidad, de modo que $\mathrm{C}^{\alpha_{1}}\left(\mathrm{~m}^{\alpha}{ }_{1}\right), \mathrm{C}^{\alpha_{2}}\left(\mathrm{~m}^{\alpha}{ }_{2}\right), \mathrm{C}^{\alpha_{3}}\left(\mathrm{~m}^{\alpha}{ }_{3}\right), \ldots, \mathrm{C}^{\alpha}\left(\mathrm{m}^{\alpha}\right)\left(\mathrm{t}_{1}\right)$ explica $\mathrm{C}^{\beta_{1}},\left(\mathrm{~s}^{\beta_{1}}{ }^{\prime}\right)\left(\mathrm{t}_{2}\right)$, entonces hay una sola y misma relación de construcción unificada, que respalda tal vía explicativa, tanto horizontalmente extendida a través del tiempo como verticalmente extendida a través de niveles ontológicos, que se despliega desde $C^{\alpha_{1}}\left(f^{\alpha_{1}}\right), C^{\alpha_{2}}\left(f^{\alpha_{2}}\right), C^{\alpha_{3}}\left(f^{\alpha_{3}}\right), \ldots, C^{\alpha_{n}}\left(f_{n}{ }_{n}\right)\left(t_{1}\right)$ a $\mathrm{C}^{\alpha_{1+}}\left(\mathrm{m}^{\alpha_{1+}}\right), \mathrm{C}^{\alpha_{2+}}\left(\mathrm{m}^{\alpha_{2+}}\right), \mathrm{C}^{\alpha_{3+}}\left(\mathrm{m}^{\alpha_{3+}}\right), \ldots, \mathrm{C}_{n+}\left(\mathrm{m}^{\alpha_{n+}}\right)\left(\mathrm{t}_{2}\right)$ en la porción $(\Psi)$ de un $\alpha$-nivel más fundamental de la realidad hasta $\mathrm{C}^{\beta_{1 *}}\left(\mathrm{~s}^{\beta_{1 *}}\right)\left(\mathrm{t}_{2}\right)$ en la porción $(\Psi ')$ de un $\beta$-nivel menos funda- 
mental de la realidad, de modo que $\mathrm{C}^{\alpha_{1}}\left(\mathrm{~m}^{\alpha}{ }_{1}\right), \mathrm{C}^{\alpha_{2}}\left(\mathrm{~m}^{\alpha}{ }_{2}\right), \mathrm{C}^{\alpha_{3}}\left(\mathrm{~m}^{\alpha}{ }_{3}\right), \ldots, \mathrm{C}^{\alpha}{ }_{n}\left(\mathrm{~m}^{\alpha}{ }_{n}\right)\left(\mathrm{t}_{1}\right)$ construye $\mathrm{C}_{1}{ }_{1},\left(\mathrm{~s}_{1}{ }_{1},\right)\left(\mathrm{t}_{2}\right)$. La fórmula ahora sería: una explicación, una relación de construcción.

De este modo podemos redefinir al panpsiquismo de construcción incluyendo la referencia a la explicación:

- (A3) Panpsiquismo de construcción $=_{\mathrm{df}}$ la conciencia de un sujeto de experiencia dependiente está construida a partir de, y explicada por, el arreglo dinámico colectivo, tanto horizontalmente extendido a través del tiempo como verticalmente extendido a través de niveles ontológicos, de las propiedades fenoménicas de un número $n$ de entidades fundamentales microfísicas.

A esta altura podemos evaluar la solución al problema de la combinación propuesto. Podemos preguntarnos ¿por qué ese arreglo dinámico colectivo, tanto horizontalmente extendido a través del tiempo como verticalmente extendido a través de niveles ontológicos, de las propiedades fenoménicas de un número $n$ de entidades fundamentales microfísicas construye un sujeto de experiencia dependiente, en lugar de nada?

Para esto, tengamos en cuenta que el panpsiquismo de construcción es una tesis explicativa. Parte del rol de una explicación es establecer patrones explicativos (Schaffer 2018, 306 y Dasgupta 2014, 569-571), es decir, las explicaciones buscan establecer abstracciones que permitan subsumir casos particulares bajo un patrón explicativo más general. Una explicación debe tener los recursos suficientes para explicar no solo los casos particulares sino, también, producir abstracciones que permitan establecer patrones explicativos más generales.

Podemos considerar un sujeto de experiencia dependiente cualquiera y es seguro que será construido a partir de cierto arreglo dinámico colectivo. Podemos considerar, a continuación, otros sujetos de experiencia dependientes y es seguro que encontraremos algún otro arreglo dinámico colectivo. Entonces hay un patrón, un patrón explicativo. Pero ¿por qué? Podemos preguntarnos si el arreglo dinámico colectivo propuesto, por sí solo, posee la suficiente fuerza explicativa como para establecer un patrón explicativo general.

No hay que olvidar que, en última instancia, el arreglo dinámico colectivo propuesto es un arreglo dinámico colectivo de sujetos de experiencia fundamentales. Ello quiere decir que son un locus interno de experiencia esencialmente para sí mismos. Son un punto de vista, y todo punto de vista excluye los demás puntos de vista (Coleman (2014)). Así, un sujeto de experiencia dependiente estará construido en el arreglo dinámico colectivo de un número $n$ de puntos de vista excluyentes de los demás puntos de vista. Es decir, paradójicamente, queremos solucionar el problema de la combinación desde entidades que en su misma naturaleza pareciesen ser excluyentes las unas de las otras, que no parecen combinables por sí solas en tanto que se agotan enteramente en ser para sí mismas.

En vista a lo anterior, entonces, el arreglo dinámico colectivo propuesto, por sí solo, no construye y explica un sujeto de experiencia dependiente porque "no hace mención" a este último. Con lo cual, parece bastante sorprendente la construcción de un sujeto de experiencia dependiente desde, solamente, sujetos de experiencia fundamentales.

Entonces parece difícil que, por sí solo, un arreglo dinámico colectivo de sujetos de experiencia fundamentales posea la suficiente fuerza explicativa como para establecer un 
patrón explicativo general que permita subsumir casos particulares bajo éste. Es necesario algo más, y es por ello que postularemos principios generales de construcción.

La postulación de principios generales de construcción no debe considerarse como arbitraria. Guarda relación con lo establecido anteriormente. Toda explicación posee una estructura tripartita (Schaffer 2017, 3), es decir, una explicación se compone de fuentes (sources), vínculos (links) y resultados (result). Por ejemplo, explicaciones que supuestamente se considerarían típicamente causales poseerían la siguiente estructura tripartita: causa, leyes de la naturaleza, efecto. O la estructura tripartita de las explicaciones que supuestamente se considerarían típicamente metafísicas se constituye de: el fundamento, leyes de la metafísica, lo fundado. Inclusive, la explicación lógica posee la misma estructura con sus premisas, reglas de inferencia y conclusión. De modo que, en tanto tesis explicativa, la ulterior estructura tripartita del panpsiquismo de construcción es: entidad constructora (sujetos de experiencia fundamentales), principios generales de construcción, y entidad construida (sujeto de experiencia dependiente). Por lo tanto, la postulación de ciertos principios generales de construcción no es arbitraria.

Los principios generales de construcción son generalizaciones no-accidentales que sostienen la vinculación entre las condiciones actualmente ocurrentes (las entidades constructoras) y las condiciones resultantes (las entidades construidas). Estos principios guían la construcción de la segunda desde la primera, lo cual da como resultado un patrón explicativo general que permite subsumir casos particulares bajo éstos. La idea es que principios generales de construcción entre entidades constructoras y entidades construidas son necesarios para explicar por qué una relación de construcción se sostiene entre estas entidades. Estos principios poseerían las siguientes características:

- Deben ser principios de evolución temporal, especificarán cómo el estado de una entidad construida evolucionará desde el estado inicial de una entidad constructora. ( $c f r$. Maudlin 2007, 172).

- Especificarán a las entidades constructoras y las reglas para su combinación. Determinan cómo se generan las entidades construidas a partir de las entidades constructoras. (cfr. Rosen 2006, 35).

En el caso del panpsiquismo de construcción, la adición de principios generales de construcción está justificada en vista a la naturaleza excluyente de los sujetos de experiencia fundamentales. No es suficiente con éstos para entender por qué hay un patrón explicativo general, que revela cómo se conectan los sujetos de experiencia fundamentales con los sujetos de experiencia dependientes. Así, al momento de considerar a los segundos, es gracias a estos principios que es seguro que será construido en el arreglo dinámico colectivo de sujetos de experiencia fundamentales. Cada caso particular que consideremos será subsumido bajo el patrón explicativo sostenido gracias a los principios generales de construcción.

Sin principios generales de construcción nada conecta a los sujetos de experiencia fundamentales con los sujetos de experiencia dependientes, ningún patrón general de construcción es revelado. En ausencia de estos principios los anteriores son solo dos cosas desconectadas. Es en presencia de los principios generales de construcción que los 
sujetos de experiencia fundamentales se conectan (construyen y explican) a los sujetos de experiencia dependientes. La conexión entre los primeros y los segundos es asegurada por medio de los principios generales de construcción.

De este modo podemos volver a redefinir al panpsiquismo de construcción incluyendo la referencia a los principios generales de construcción:

- (A4) Panpsiquismo de construcción = df la conciencia de un sujeto de experiencia dependiente está construida a partir del arreglo dinámico colectivo, tanto horizontalmente extendido a través del tiempo como verticalmente extendido a través de niveles ontológicos, de las propiedades fenoménicas de un número $n$ de entidades fundamentales microfísicas más principios generales de construcción que vinculan el arreglo dinámico colectivo, tanto horizontalmente extendido a través del tiempo como verticalmente extendido a través de niveles ontológicos, de las propiedades fenoménicas de un número $n$ de entidades fundamentales microfísicas a un sujeto de experiencia dependiente.

\section{VII}

Finalmente, podemos considerar que, con relación a los otros panpsiquismos, el panpsiquismo de construcción tiene mayor mérito. Por un lado, dada la identidad entre fundación y causalidad, la distinción entre estos dos panpsiquismos es falsa. Como decíamos anteriormente, no hay dos relaciones de dependencia ontológica diferentes en virtud de las cuales el panpsiquismo constitutivo y el panpsiquismo emergentista sean dos tesis diferentes. Es importante remarcar que lo que proponemos no es una cuestión meramente terminológica. No se trata de que ahora llamemos simplemente "panpsiquismo de construcción" a lo que antes llamábamos con dos nombres distintos, "panpsiquismo constitutivo" y "panpsiquismo emergentista". De lo que se trata es de cómo es la realidad para que los anteriores panpsiquismos sean distinciones adecuadas o inadecuadas. ${ }^{8}$ La cuestión terminológica es una consecuencia de lo anterior. Así, para el caso del panpsiquismo de construcción hay una sola y misma relación de dependencia ontológica unificada.

Por el otro, téngase en cuenta que el espíritu del panpsiquismo es de continuidad y uniformidad, y que de la relación de construcción se deriva la estructura metafísica de la realidad. Considérese la asimetría de la relación de construcción, según la cual el relatum de un lado de la relación de construcción está construido por el otro relatum y no viceversa. Esto impone un orden metafísico entre los contenidos de la realidad. Entonces, al momento en que el hecho $x$ construye el hecho $y$, el hecho $x$ se encuentra del lado relativamente más fundamental de la relación de construcción y el hecho $y$ del lado relativamente menos fundamental. Esto nos muestra la estructura metafísica de la realidad. Así, es el ordenamiento impuesto por la relación de construcción, que conecta el hecho $x$ (en un nivel más profundo de la realidad) al hecho $y$ (en un nivel más superficial de la realidad), el que construye la estructura metafísica de la realidad. La estructura metafísica de la realidad se construye a partir de la relación de construcción y la relación de construcción es unificada, no varía, por lo tanto, la estructura metafísica de la realidad también es unificada.

8 "Panpsiquismo constitutivo" y "panpsiquismo emergentista" tampoco pueden ligarse a modo de género y especie con la relación de construcción porque ello acarrea el problema de la parsimonia conceptual esbozado anteriormente. 
Así el panpsiquismo de construcción ofrece una solución al problema de la combinación proponiendo una sola y misma relación de dependencia ontológica unificada, de la cual se deriva una sola y misma estructura metafísica unificada de la realidad para las diferentes entidades en distintos tiempos y niveles ontológicos. El panpsiquismo apela a la continuidad y uniformidad, y el panpsiquismo de construcción con su solución mantiene esa continuidad y uniformidad. En cambio, tanto panpsiquismo constitutivo como panpsiquismo emergentista rompen con el espíritu del panpsiquismo porque, si fuesen distinciones adecuadas, sus soluciones al problema de la combinación ofrecen dos relaciones distintas (fundación y causalidad), de las cuales se deriva una estructura metafísica de la realidad desunida. La estructura metafísica de la realidad derivada de la fundación que impone un orden a diferentes entidades en distintos niveles ontológicos difiere de la estructura metafísica de la realidad derivada de la causalidad que impone un orden a diferentes entidades en distintos tiempos. Es más, al ser diferentes las soluciones de estos panpsiquismos no son necesariamente convergentes; puede ocurrir que la solución al problema de la combinación se resuelva en términos de la relación de fundación y la solución al problema de la interacción mente/cuerpo sea causal. Esto rompe con la continuidad y uniformidad que proponía el panpsiquismo. ¿Por qué apelar a soluciones del problema de la combinación que rompen con el espíritu del panpsiquismo cuando se dispone de una solución que lo conserva?

Por último, quisiéramos remarcar que es la relación de construcción, y su estructura metafísica unificada derivada, la base de la posibilidad de solución al problema de la combinación y desde el panpsiquismo de construcción esta unificación parece mucho más sencilla: “(1) tomar los hechos (...) de un sistema dado, (2) aplicar la teoría (...) a estos hechos, y así (3) derivar una caracterización precisa de las experiencias asociadas que la teoría predice." (Chalmers 1997, 420). De este modo, el panpsiquismo de construcción tiene mayor mérito que las otras soluciones propuestas desde el panpsiquismo al problema de la combinación. ${ }^{9}$

\section{Bibliografía}

Alvarado, J. T. (MS), «Fundación y causalidad», <https://www.academia.edu/38243815/ Fundacio_n_y_causalidad.pdf $>$.

Bennett, K. (2011), «Construction area (no hard hat required)», Philosophical Studies, 154, pp. 79-104.

Bennett, K. (2017), Making Things Up, Oxford: Oxford University Press.

Berker, S. (2018), «The Unity of Grounding», Mind, 127 (507), pp. 729-777.

Bernstein, S. (2016), «Grounding is Not Causation», Philosophical Perspectives, 30, pp. 21-38.

Chalmers, D. (1995), «Facing up to the Problem of Consciousness», Journal of Consciousness Studies, 2 (3), pp. 200-219.

Chalmers, D. (1997), «Moving Forward on the Problem of Consciousness», en: Shear, J. (ed.): Explaining Consciousness: The 'Hard Problem', Cambridge: MIT Press, pp. 379-422.

Chalmers, D. (2006), «Strong and Weak Emergence», en: Clayton, Ph. y Davies, P. (eds.): The Re-Emergence of Emergence, Oxford: Oxford University Press, pp: 244-254.

9 Quisiera agradecer a Ángelo Briones Belmar, Gonzalo Rodriguez-Pereyra, al arbitraje anónimo de la Revista Daimon y a Yanira Karina de cuyos comentarios se ha beneficiado enormemente este trabajo. 
Chalmers, D. (2010), «Consciousness and its Place in Nature», en: Chalmers, D. (ed.): The Character of Consciousness, Oxford: Oxford University Press, pp. 103-139.

Chalmers, D. (2017), «The Combination Problem for Panpsychism», en: Brüntrup, G. y Jaskolla, L. (eds.): Panpsychism. Contemporary Perspectives, Oxford: Oxford University Press, pp: 179-214.

Coleman, S. (2014), «The Real Combination Problem: Panpsychism, Micro-Subjects, and Emergence», Erkenntnis, 79 (1), pp. 19-44.

Dasgupta, S. (2014), «The Possibility of Physicalism», Journal of Philosophy,111 (9-10), pp. 557-592.

Fine, K. (2001), «The Question of Realism», Philosophers’ Imprint, 1 (2), pp. 1-30.

García Encinas, M. J. (2018), «Causalidad», Enciclopedia de la Sociedad Española de Filosofía Analitica, <http://www.sefaweb.es/causalidad/>.

Goff, Ph. (2011), «There is no Combination Problem», en: Blamauer, M. (ed.): The Mental as Fundamental. New Perspectives on Panpsychism, Frankfurt: Ontos Verlag, pp. 131-140.

Goff, Ph. (2017), Consciousness and Fundamental Reality, New York: Oxford University Press.

Halpern, J. (2000), «Axiomatizing Causal Reasoning», Journal of Artificial Intelligence Research, 12, pp. 317-337.

Heil, J. (2015), The Universe as We Find It, Oxford: Oxford University.

Humphreys, P. (1997), «How Properties Emerge», Philosophy of Science, 64 (1), pp. 1-17.

James, W. (1983), The Principles of Psychology, Massachusetts: Harvard University Press.

Kim, J. (1994), «Explanatory Knowledge and Metaphysical Dependence», Philosophical Issues, 5, pp. 51-69.

Kim, J. (2002), «El Problema Mente-Cuerpo tras Cincuenta Años», Azafea, 4, pp. 45-63.

Kivatinos, Th.(2017), Grounding, Causation and the Unity of Ontological Structure (Tesis doctoral), $<$ https://academicworks.cuny.edu/cgi/viewcontent.cgi?article $=3429 \&$ context=gc etds $>$.

Koslicki, K. (2016), «Where Grounding and Causation Part Ways: Comments on Schaffer», Philosophical Studies, 173, pp. 101-112.

Leibniz, G. W. (1981), New Essays on Human Understanding, Cambridge: Cambridge University Press.

Levine, J. (1983), «Materialism and Qualia: The Explanatory Gap», Pacific Philosophical Quarterly, 64, pp. 354-361.

Maudlin, T. (2007), The Metaphysics Within Physics, Oxford: Oxford University.

Miguel, H. (2019), «Teorías contemporáneas de la causación», en: Vanney, C. E., Silva, I. y Franck, J. F. (eds.): Diccionario Interdisciplinar Austral, <http://dia.austral.edu.ar/ Teorías_contemporáneas_de_la_causación $>$.

Mørch, H. H. (2014), Panpsychism and Causation: A New Argument and a Solution to the Combination Problem (Tesis doctoral), <https://philpapers.org/archive/HASPAC-2.pdf $>$.

Nagel, T. (2003), «¿Cómo es ser un murciélago?» en: Ezcurdia, M. y Hansberg, O. (comps.): La Naturaleza de la Experiencia. Volumen I: Sensaciones, México, D.F.: Universidad Nacional Autónoma de México, pp. 45-63.

Pearl, J. (2009), Causality: Models, Reasoning, and Inference (Second Edition), Cambridge: Cambridge University Press. 
Raven, M. J. (2015), «Ground», Philosophy Compass, 10 (5), pp. 322-333.

Roelofs, L (2014), «Phenomenal Blending and the Palette Problem», Thought: A Journal of Philosophy, 3 (1), pp. 59-70.

Rosen, G. (2006), «The Limits of Contingency», en: MacBride, F. (ed.): Identity and Modality, Oxford: Clarendon Press, pp. 13-39.

Rosen, G. (2010), «Metaphysical Dependence: Grounding and Reduction», en: Hale, R. y Hoffman, A. (eds.): Modality: Metaphysics, Logic, and Epistemology, Oxford: Oxford University Press, pp. 109-135.

Schaffer, J. (2009), «On What Grounds What», en: Chalmers, D., Manley, D. y Wasserman, R. (eds.): Metametaphysics. New Essays on the Foundations of Ontology, Oxford: Clarendon Press, pp. 347-383.

Schaffer, J. (2016), «Grounding in the Image of Causation», Philosophical Studies, 173, pp. 49-100.

Schaffer, J. (2017), «The Ground Between the Gaps», Philosophers’ Imprint, 17 (11), pp. $1-26$.

Schaffer, J. (2018), «Laws for Metaphysical Explanation», Royal Institute of Philosophy Supplement, 82, pp. 1-22.

Seager, W. (2010), «Panpsychism, Aggregation and Combinatorial Infusion», Mind \& Matter, 8 (2), pp. 167-184.

Strawson, G. (2017), «Mind and Being. The Primacy of Panpsychism», en: Brüntrup, G. y Jaskolla, L. (eds.): Panpsychism. Contemporary Perspectives, Oxford: Oxford University Press, pp. 75-112.

van Gulick, R. (2001), «Reduction, Emergence and Other Recent Options on the Mind/Body Problem. A Philosophic Overview», Philosophical Quarterly, 37 (148), pp. 253-277.

van Inwagen, P. (1990), Material Beings. Ithaca: Cornell.

Wilhelm, I. (2020), «Explanatory Priority Monism», Philosophical Studies. doi: 10.1007/ s11098-020-01478-z

Wilson, A. (2018), «Metaphysical Causation», Noûs, 52 (4), pp. 723-751.

Wilson, A. (MS), «Classifying Dependencies», <http://alastairwilson.org/files/cdweb.pdf>.

Wilson, J. (2014), «No Work for a Theory of Grounding», Inquiry, 57 (5-6), pp. 1-45.

Wilson, J. (2016), «Metaphysical Emergence: Weak and Strong», en: Bigaj, T. y Wüthrich, Ch. (eds.): Metaphysics in Contemporary Physics, Amsterdam/New York: Rodopi Brill, pp. 345-402. 